\title{
Effect of paratuberculosis on slaughter weight and slaughter value of dairy cows
}

\author{
A. B. Kudahl*1 and S. S. Nielsent \\ ${ }^{*}$ Faculty of Agricultural Sciences, Department of Animal Health, Welfare and Nutrition, University of Aarhus, Blichers Allé 20, PO Box 50, \\ DK-8830 Tjele, Denmark \\ †Faculty of Life Sciences, Department of Large Animal Sciences, University of Copenhagen, Grønnegårdsvej 8, DK-1870 Frederiksberg, \\ Denmark
}

\section{ABSTRACT}

The effect of infection with Mycobacterium avium ssp. paratuberculosis (MAP) on slaughter weight and slaughter value of dairy cows was evaluated. Two data sets were analyzed: 1) recordings from 1,031 cows from herds in a pilot study to control MAP infections, and 2) recordings from 36,455 cows from herds participating in the Danish MAP control program. The effect of stage of MAP infection on carcass weight and slaughter value was assessed by ANOVA. Infection stage was diagnosed by repeated milk antibody ELISA in both data sets. Furthermore, repeated fecal culture was recorded in data set 1 and occurrence of enteritis or enteric edema found at slaughter was recorded in data sets 1 and 2 . Compared with presumably unaffected cows with at least 2 ELISA negative tests, slaughter weight and value were reduced by 10 and $17 \%$, respectively, in cows with positive ELISA at slaughter. If the cow was also positive using fecal culture, slaughter weight and value were reduced up to 15 and $31 \%$, respectively. The slaughter weight and value were reduced an additional 20 and $31 \%$, respectively, for cases with recorded enteritis or edema. Thereby, summarized weight losses of up to $31 \%$ and slaughter value losses up to $48 \%$ occurred. Cows with negative fecal cultures had reduced slaughter results only if they were ELISA-positive in the last 2 tests. Losses of both slaughter weight and slaughter value caused by MAP were more severe than previously estimated. These losses could be predicted by repeated milk ELISA tests with or without confirmation with fecal culture.

Key words: antibody profile, Mycobacterium avium ssp. paratuberculosis, slaughter weight, slaughter value

Received January 16, 2009

Accepted May 26, 2009.

${ }^{1}$ Corresponding author: Anneb.Kudahl@agrsci.dk

\section{INTRODUCTION}

Infection with Mycobacterium avium ssp. paratuberculosis (MAP) in cattle can cause reduction in milk production and weight loss in the clinical stage of infection (Chiodini et al. 1984). Infections with MAP are usually assumed to occur during the first months of life. Calves can be infected through oral ingestion of MAP in feces or milk from adult cattle and the infection may develop into chronic enteritis over the following years (Manning and Collins, 2001). Cows infected with MAP eventually experience declining milk yield, intermittent diarrhea, weight loss, and terminally devastating mortal diarrhea (Chiodini et al., 1984). Therefore, MAP can cause losses to the farmer because both milk yield and slaughter weight are reduced. Furthermore, cow mortality can be higher in MAP-infected herds, depending on the culling strategy of the farmer (Groenendaal et al., 2002; Kudahl et al., 2007).

Losses at slaughter associated with MAP infection have only been quantified in a few previous studies, with reduction of slaughter weight reported in some and reduction in slaughter value in others. In addition to the weight of the animal at time of slaughter, slaughter value also includes the age of the animal and the price per kilogram, reflecting the quality of the meat. Slaughter weight has been reported to be reduced by $10 \%$ in carcasses in which ileocecal lymph nodes, ileum, rectum, or feces were culture positive for MAP compared with culture-negative cows (Whitlock et al., 1985). Slaughter value has been reported to be reduced by $30 \%$ in cows with clinical symptoms (Benedictus et al., 1987). Not all MAP-infected animals experience reduced weight because of the slow development of MAP infection; for example, McKenna et al. (2004) found no relationship between BCS and occurrence of MAP in the intestines or lymph nodes. Only animals that are unable to control the infection are expected to experience reduced weight caused by MAP infection. Loss of control is associated with occurrence of antibody-mediated immune reactions, which indicates progression of the infection (Coussens, 2001). However, antibody levels may fluctu- 
Table 1. Definition of antibody groups based on results of milk antibody ELISA repeated every third month

\begin{tabular}{ll}
\hline Antibody group & Definition of antibody group \\
\hline A0 & A minimum of two available tests, all tests negative \\
A1 & Only one available test, which was negative \\
A2 & Last three tests negative, but previously at least one positive test \\
A3 & Response fluctuating between negative and positive, last test negative \\
A4 & Last test positive, but previous tests were all negative \\
A5 & Last two or more tests positive \\
\hline
\end{tabular}

ate in both milk (Nielsen, 2008) and serum (Sweeney et al., 2006) in the transitional phase between control and loss of control. Animals with fluctuating antibody levels have been demonstrated to have higher milk yield than their test-negative herd-mates, whereas animals with a persistent occurrence of antibodies can experience reduced milk yield (Nielsen et al., 2009). Our hypothesis was that specific MAP antibody profiles in a similar way are associated with reduced weight and value at slaughter.

The aim of this study was to assess the association between slaughter weight and value and MAP infection stage. Infection stages of MAP were estimated based on the repeated milk antibody ELISA (MAP antibody profile) alone or in combination with a) fecal culture results, or b) findings of enteritis or edema at slaughter as indicators of clinical disease.

\section{MATERIALS AND METHODS}

\section{Animals, Herds, and Records}

The study included 2 study populations, which were analyzed separately because the diagnostic information differed between the 2 data sets. The first data set was sampled in the period from August 1999 to March 2003, and included 1,031 Danish Holstein (Black and White) cows from 7 herds. The herds also included 334 Jerseys, 103 crossbreds, 31 Danish Holstein (Red and White), and 10 Red Danish cows, but these animals were not included in the analyses because of their small numbers. Milk samples were obtained from all lactating cows at the monthly routine test-day recordings and repeated 2 to 36 times per cow with a median of 9 and a mean of 11 times per cow. Feces was sampled 4 times per year per herd, and resulted in 1 to 23 samples for each cow with a mean of 5.3 and a median of 4 samples per cow.

The second data set was based on recordings from the Danish national voluntary control program on paratuberculosis (Nielsen, 2007) from February 2006 to December 2007, and from pilot herds used to establish the program (2003-2005). Milk samples were obtained from all lactating cows in each herd every third month.
Milk sampling was repeated 1 to 24 times with a mean of 2.4 and a median of 2 times per cow. No fecal samples were obtained from animals in data set 2 . In total, data from 28,733 Danish Holstein cows in 893 herds and 7,722 Jersey cows from 239 herds were included in data set 2 .

\section{Diagnostic Tests}

Milk samples were tested with a MAP-specific antibody ELISA (Nielsen, 2002) and fecal samples were cultured using Herrold's egg yolk medium as described in Nielsen et al. (2004). Milk samples were defined as positive whenever corrected optical density (OD) values (i.e., the $\mathrm{OD}$ of a sample minus the $\mathrm{OD}$ of a negative control OD) exceeded 0.3. Subsequently, cows were divided into 5 different antibody groups based on the last 4 ELISA results (Table 1). These groups were assumed to reflect different stages of infection and have been described to be associated with differences in detectable MAP shedding and milk production (Nielsen, 2008; Nielsen et al., 2009). Groups A0 and A2 have a low risk of detectable bacterial shedding, group A3 has an intermediate risk, whereas groups A4 and A5 have a high risk (Nielsen, 2008). Groups A4 and A5 are infection stages associated with decreased milk production, whereas cows in groups A2 and A3 are infection stages associated with increased milk yield, all compared with milk yield of cows in group A0. The distribution of cows in different antibody groups is listed in Table 2. Cows with only one ELISA test result (group A1) were excluded from further analyses because of the limited information they provided.

A cow was defined to be fecal culture (FC) positive if one or more fecal samples were positive.

\section{Slaughter Recordings}

Data on carcass weight, muscle mass classification (based on the EUROP system; European Union, 2004), and registrations of signs of diseases or injuries on the carcass were obtained from Danish slaughterhouses, which make these registrations routinely. Based on these data, cows were categorized into 5 groups: 1) 
Table 2. Number of cows in the 5 antibody groups used in the analysis ${ }^{1}$

\begin{tabular}{|c|c|c|c|c|c|c|c|}
\hline Data set & Breed & Fecal culture & \multicolumn{5}{|c|}{ Antibody group } \\
\hline 1 & Danish Holstein & Positive & $48(0)$ & $3(0)$ & $13(0)$ & $27(0)$ & $94(0)$ \\
\hline 2 & Danish Holstein & Unknown & $17,486(3)$ & $307(0)$ & $4,708(20)$ & $2,557(9)$ & $3,675(26)$ \\
\hline 2 & Jersey & Unknown & $4,146(14)$ & $81(0)$ & $1,458(9)$ & $669(1)$ & $1,368(13)$ \\
\hline
\end{tabular}

${ }^{1}$ Numbers in parentheses are the number of cows in the group where enteritis and/or edema were found in the carcass at slaughter. These diagnoses only occurred in data set 2 .

nonspecific recordings; 2) physical injuries; 3) infections without enteric edema and enteritis; 4) enteric edema or enteritis; and 5) no signs of disease. Cows with recorded physical injuries on the carcass were excluded to avoid bias from other factors causing a potential weight loss.

Information on age, calving number, breed, lactation stage, and cause of death (dead or slaughtered) were obtained from the Danish Cattle Database (Danish Cattle Federation, 2006).

\section{Statistical Analysis}

Two similar statistical models were analyzed, one with slaughter weight as outcome and the other with slaughter value as outcome. The slaughter value was calculated as slaughter weight multiplied by the price per kilogram. The prices per kilogram originated from the official EUROP quotation on May 14, 2008, from the Danish slaughterhouses. Prices according to age, total slaughter weight, and EUROP classification are listed in Table 3. A complete set of prices from the same date was chosen to avoid bias from prices fluctuating over time, which would make slaughter values incomparable. The effect of MAP infection status (based on ELISA, fecal culture, and enteritis/enteric edema) on slaughter weight and slaughter value was analyzed by ANOVA. The following initial model was set up using a random coefficient mixed model with 2 levels:

$$
\begin{aligned}
\mathrm{Y}_{j k l m n o}= & \beta_{0}+\beta_{1 l}+\beta_{2 m}+\beta_{3} \mathrm{DP}_{j k}+\beta_{4 n}+\beta_{5 o} \\
& +\beta_{6} \mathrm{DIM}_{j k}+\mathrm{H}_{k}+\mathrm{e}_{i j k l m n o}
\end{aligned}
$$

where $\mathrm{Y}_{j k l m n o}=$ slaughter weight or slaughter value; $\mathrm{DP}_{j k}=$ days passed since first positive ELISA and until slaughter of the $j$ th cow of the $k$ th herd; $\operatorname{DIM}_{j k}=$ days in milk at slaughter of the $j$ th cow of the $k$ th herd; $\beta_{0}$ $=$ overall mean of either the slaughter weight or slaughter value; $\beta_{1 l}=$ fixed effect of the $l$ th antibody group, where $l=\mathrm{A} 0, \mathrm{~A} 2, \mathrm{~A} 3, \mathrm{~A} 4$, or $\mathrm{A} 5 ; \beta_{2 m}=$ fixed effect of the $m$ th fecal culture response where $m=\mathrm{FC}-$ or $\mathrm{FC}+; \beta_{3}=$ fixed linear regression coefficient of $\mathrm{DP} ; \beta_{4 n}$ $=$ fixed effect of category $n$ of symptoms of chronic diseases found on the carcass; $\beta_{50}=$ fixed effect of the oth parity (categories 1,2 , and $3+$ ); $\beta_{6}=$ fixed linear regression coefficient of DIM; $\mathrm{H}_{k}=$ random intercept of the $k$ th herd; and $\mathrm{e}_{j k l m n o}=$ a random residual component assumed independent, identically distributed normal, $\mathrm{N}\left(0, \sigma^{2}\right)$.

Parity and lactation stage (DIM) were included in the model as an alternative to age, because weight is known to be associated with lactation stage: The first 5 to 10 wk after calving a negative energy balance often causes weight loss followed by a 50 - to $80-\mathrm{kg}$ weight gain during the rest of the lactation. Climatic stress factors or feed rations could result in variation by year and season in some areas. However, under Danish conditions the seasonal factor was expected to be of little relevance

Table 3. Prices used for calculation of slaughter value ${ }^{1}$

\begin{tabular}{llcc}
\hline & & & \multicolumn{2}{c}{ Price $^{1}(\mathrm{US} \$ / \mathrm{kg})$} \\
\cline { 3 - 4 } Age $(\mathrm{mo})$ & Slaughter weight $\mathrm{W}(\mathrm{kg})$ & Muscle mass index $\{1,2,3\}$ & Muscle mass index $\{4,5\}$ \\
\hline$<42$ & $\mathrm{~W}>230$ & Same as age $>42$ (see below) & 5.24 \\
$<42$ & $\mathrm{~W} \leq 230$ & Same as age $>42$ (see below) & 4.14 \\
$>42$ & $\mathrm{~W}>280$ & 3.68 & 4.18 \\
$>42$ & $260<\mathrm{W} \leq 280$ & 3.32 & 3.68 \\
$>42$ & $240<\mathrm{W} \leq 260$ & 3.04 & 3.40 \\
$>42$ & $220<\mathrm{W} \leq 240$ & 2.88 & 3.11 \\
$>42$ & $180<\mathrm{W} \leq 220$ & 2.73 & 2.96 \\
$>42$ & $\mathrm{~W} \leq 180$ & 2.58 & 2.81 \\
\hline
\end{tabular}

${ }^{1}$ The prices are classified according to age of the slaughtered animal, slaughter weight, and quality [muscle mass index (form)]. 
Table 4. Reduction of slaughter weight $(\%)$ in cows of various antibody groups ${ }^{1}$

\begin{tabular}{|c|c|c|c|c|c|c|c|c|}
\hline Data set & Breed & Fecal culture & \multicolumn{5}{|c|}{ Antibody group } & $\begin{array}{l}\text { Enteritis or } \\
\text { enteric edema }\end{array}$ \\
\hline 1 & Danish Holstein & Positive & -2.6 & -6.1 & $-11.9^{* *}$ & $-14.9^{* *}$ & $-15.6^{* * *}$ & \\
\hline 2 & Danish Holstein & Unknown & 0.0 & 1.9 & $-3.8^{* * *}$ & $-7.3^{* * *}$ & $-10.1^{* * *}$ & $-19.5^{* * *}$ \\
\hline 2 & Jersey & Unknown & 0.0 & -1.2 & -3.8 & $-7.1^{* * *}$ & $-10.1^{* * *}$ & $-20.0^{* * *}$ \\
\hline
\end{tabular}

${ }^{1}$ For cows with findings of enteritis or enteric edema at slaughter, the losses in the last column should be added to losses given for the antibody group. Enteritis or enteric edema were also registered in data set 1, but with zero occurrences. $P$-values refer to the difference compared with antibody group 0 (and fecal culture negative in data set 1).

** $P<0.01 ; * * * P<0.001$.

because most cows have an ad libitum full feed ration and are kept indoors all year round. Therefore, year and season were not included in the analyses.

The full model was reduced by backward elimination. Data set 1 was only analyzed for Danish Holstein, and data set 2 was analyzed for both Jerseys and Danish Holstein. Both data sets were analyzed first with slaughter weight as the outcome variable, and then with slaughter value as the outcome variable. The diagnostic information included in the model was antibody group at last sampling before slaughter, fecal culture (only data set 1), and period from first positive ELISA until slaughter (number of days). The last factor was included because we hypothesized that the weight loss would increase the longer the ELISA-positive cows were kept in the herd. All possible combinations of first-order interactions between all fixed effects were included initially besides the main effects.

Mixed effects ANOVA was performed using the Mixed procedure in SAS version 8.2 (Littell et al., 2006). Models were reduced by backward elimination using the likelihood-ratio test at the $5 \%$ level as the cut-off for inclusion in the model. Given that the interaction between $\beta_{1 l}$ and $\beta_{2 m}$ was significant, $\beta_{1 l}$ was nested in $\beta_{2 m}$. Data set 2 was analyzed separately for Danish Holstein and Danish Jerseys.

\section{RESULTS}

The reduction in slaughter weight of cows with various antibody profiles compared with the slaughter weight of antibody-negative cows is listed in Table 4 . The last column shows the estimates of the additional effect of enteritis or edema on the slaughter weight in data set 2. This loss should be added to the loss associated to the antibody group, because there was no interaction between these variables. This means that a Danish Holstein cow in group A5 with enteritis or edema at slaughter would have a total reduction of slaughter weight of $30 \%$. There were zero occurrences of enteritis and edema in data set 1.

Reduction in slaughter value related to the various MAP infection stages are summarized in Table 5. The table is equivalent to Table 4, except that the loss in monetary value reflects not only a lower weight but also a lower price per kilogram due to age (younger/ older than $42 \mathrm{mo}$ ) and lower meat content and quality of MAP-infected slaughter cows.

The final models with estimates of all fixed effects on slaughter weight and slaughter value of data set 1 are presented in Appendix Table A1 and Table A2, respectively. Weight at slaughter was not only affected by MAP infection, but also by lactation stage $(0.10 \mathrm{~kg} / \mathrm{d})$

Table 5. Reduction of slaughter value (\%) in cows of various antibody groups ${ }^{1}$

\begin{tabular}{lllcccccc}
\hline & & \multicolumn{5}{c}{ Antibody group } \\
\cline { 3 - 7 } Data set & Breed & Fecal culture & A0 & A2 & A3 & A4 & A5 & enteritis or \\
\hline 1 & Danish Holstein & Positive & -6.5 & -23.2 & $-28.6^{*}$ & $-23.8^{* *}$ & $-30.8^{* * *}$ \\
1 & Danish Holstein & Negative & 0 & -1.3 & 0.7 & -1.6 & -7.0 \\
2 & Danish Holstein & Unknown & 0 & -3.9 & $-12.9^{* * *}$ & $-7.9^{* * *}$ & $-16.6^{* * *}$ & $-31.2^{* * *}$ \\
2 & Jersey & Unknown & 0 & 2.7 & $-10.5^{* * *}$ & -3.3 & $-14.4^{* * *}$ & $-28.0^{* * *}$ \\
\hline
\end{tabular}

${ }^{1}$ For cows with findings of enteritis or enteric edema at slaughter, the losses in the last column should be added to losses given for the antibody group. Enteritis or enteric edema were also registered in data set 1, but with zero occurrences. $P$-values refer to the difference compared with antibody group 0 (and fecal culture negative in data set 1 ).

${ }^{*} P<0.05 ;{ }^{* *} P<0.01 ; * * P<0.001$. 
and parity (cows slaughtered in parity 3 weighed 20.8 and $8.8 \mathrm{~kg}$ more than cows slaughtered in parities 1 and 2 , respectively). The final model of effects on slaughter value with data set 1 as an example (Table A2) lists the absolute monetary value (US\$). The basic value of a cow was US\$657 (intercept). This was for a "reference cow": a Danish Holstein of third parity or older that was FC-negative and classified in antibody group A0 (repeatedly negative) at the time of slaughter. The fixed effects of being classified in other antibody groups are listed in the "estimate" column. As an example, the value of a FC-positive cow in antibody group A5 was US\$201 less than the price of a negative reference cow.

\section{DISCUSSION}

Infections with MAP can cause reduction in slaughter weight and slaughter value, and the severity of infection correlates with the size of the reduction. Cows that were ELISA-positive with findings of enteritis or enteric edema or positive fecal culture will experience the highest losses, irrespective of the antibody profile if antibodies have previously been detected. Cows with repeated positive ELISA results (group A5) had the biggest losses compared with ELISA-negative cows (group A0), but cows with fluctuating antibody results (group A3) could also experience significant losses. This is in contrast to the losses in milk production, which have been associated only with groups A4 and A5. These data suggest that the infection develops faster in cows in group A4 compared with cows in group A3.

The losses were smaller in cows with fluctuating ELISA results in which the last test result was negative (group A3). These cows may still be in a phase where they partly control the infection. Group A2 cows, which were previously ELISA positive but negative in the last 3 tests, only had reduced slaughter value if they were FC-positive. There was a negligible difference in the slaughter weight results between Jerseys and Danish Holstein and a minor difference in slaughter value tending to a stronger effect of MAP infections on the slaughter value of Jerseys compared with Danish Holsteins.

Our results show that MAP-associated losses of slaughter value appeared to be twice the relative losses of slaughter weight. This underlines the importance of including meat quality in the estimation of the economic losses incurred to the farmer. The estimated losses described here were more severe than described in previous studies. Whitlock et al. (1985) found culture-positive nonclinical animals to have a weight loss of $10 \%$. We found that FC-positive cows that had also been ELISA-positive on one or more occasions had a slaughter weight loss of 12 to $15 \%$ and slaughter value losses of up to $31 \%$. Findings on the carcass at slaughter suggesting that the cow had been in a clinical phase of infection (i.e., enteritis or enteric edema) resulted in an additional loss of $20 \%$ of slaughter weight and approximately $30 \%$ of the slaughter value. Benedictus et al. (1987) estimated a total loss of slaughter value among clinical cows of $30 \%$. In the worst case, our estimates could reach a total loss of $48 \%$ ( $17 \%$ loss due to being in antibody group A5 with unknown fecal culture and $31 \%$ due to clinical signs).

The number of days from the first positive ELISA to slaughter was included in the initial models because we hypothesized that weight loss would increase when this period was prolonged. Surprisingly, a tendency to increased slaughter weight within this period was seen. However, the effect was insignificant and therefore excluded from the model. This positive tendency can be a result of farmers' slaughter strategies: test-positive cows that show signs of weight loss are slaughtered immediately, whereas positive cows able to control the infection without weight loss are kept in the herd for a longer period.

Repeated milk ELISA testing gave a good prediction of potential losses in slaughter results. However, when combined with fecal culture the prediction was even better. If the animal was positive, not only in ELISA but also in fecal culture, there was a risk of even larger loss in slaughter results. If a cow was culture-negative, the risk of weight loss was small unless the last 2 ELISA results were positive (group A5). Despite differences in sampling intervals between the 2 data sets, the reductions in slaughter weight and values had a similar pattern for the different antibody groups between the 2 models.

The perspective for the farmers of using the regular milk ELISA tests is thus to focus attention on animals that have had their first positive ELISA. However, based on our results, slaughter decisions should only be taken if the animal has had 2 or more consecutive positive ELISA results. These cows have a higher risk of a reduced slaughter weight and reduced milk yield (Nielsen et al., 2009) and a higher risk of being infectious (Nielsen, 2008). If the farmer wants confirmation of the diagnosis before slaughter, the ELISA can be supplemented with a fecal culture test. A disadvantage is that it may take 2 to 3 mo to obtain the test result. Simulation studies have suggested that confirmation of the diagnosis using fecal culture is unprofitable $(\mathrm{Ku}-$ dahl et al., 2007). Slaughter value is only one of several considerations to take when deciding the optimal time for slaughtering a MAP-infected animal. In addition, the farmer has to consider 1) the actual milk yield of the infected cow compared with the replacement animal; 2) price and availability of replacement animals; 
and 3) infectiousness of the infected animal. Simulation models can be effective tools to support these complex decisions. A herd simulation study conducted with the PTB-Simherd model suggested that immediate slaughtering of cows in group A5 was only economically optimal if reproduction in the herd was good (heat observation rate of $60 \%$ ) and if infection routes were not closed properly (Kudahl et al., 2008). In case of poor reproduction (heat observation rate of $40 \%$ ) or closed infection routes, there was a minor economic benefit from keeping these cows until milk yield was reduced to the general limit for slaughtering low-yielding cows. Cows of group A5 in the Danish MAP control program are recommended to be slaughtered within the current lactation and never inseminated again to limit their contamination of the farm environment with MAP (Nielsen, 2007).

\section{CONCLUSIONS}

Losses in slaughter weight and value caused by MAP were more severe than estimated in previous studies. Slaughter weight and value were reduced by 10 and $17 \%$, respectively, for ELISA-positive cows compared with presumably unaffected test-negative cows. Slaughter weight and value were reduced 15 and $31 \%$, respectively, for cows testing positive by fecal culture. Our results showed that losses in slaughter weight and slaughter value could be predicted by repeated milk ELISA tests with or without confirmation with a fecal culture.

Farmers should pay increased attention to animals that have had their first positive ELISA, but the decision on slaughtering an apparently unaffected cow could be postponed until the animal has had at least 2 positive tests in a row. Cows with repeated positive ELISA results have a high risk of shedding MAP and of having a reduced milk yield in addition to the potential reduction in slaughter weight and value.

\section{ACKNOWLEDGMENTS}

This study was supported by the Danish Directorate of Food, Fisheries and Agro Business (no. 3412-0601550), the Danish Milk Levy Fund, and the Danish Cattle Levy Fund. The Danish Cattle Federation is thanked for providing access to the data.

\section{REFERENCES}

Benedictus, G., A. A. Dijkhuizen, and J. Stelwagen. 1987. Economic losses due to paratuberculosis in dairy cattle. Vet. Rec. 121:142146.

Chiodini, R. J., H. J. van Kruiningen, and R. S. Merkal. 1984. Ruminant paratuberculosis (Johne's disease): The current status and future prospects. Cornell Vet. 74:218-262.

Coussens, P. M. 2001. Mycobacterium paratuberculosis and the bovine immune system. Anim. Health Res. Rev. 2:141-161.

Danish Cattle Federation. 2006. Principles of Danish Cattle Breeding. http://www.lr.dk/kvaeg/diverse/principles.pdf

European Union. 2004. European Union legislation. Order 882/2004: Technical norms for bovine carcass classification http://www. europ.ro/english/legislation_en/ENTechnical\%20Norms\%20 Bovine\%20Order\%20882.doc Accessed December 4, 2008.

Groenendaal, H., M. Nielen, A. W. Jalvingh, S. H. Horst, D. T. Galligan, and J. W. Hesselink. 2002. A simulation of Johne's disease control. Prev. Vet. Med. 54:225-245.

Kudahl, A. B., S. S. Nielsen, and S. Østergaard. 2008. Economy, efficacy and feasibility of a risk-based control program against paratuberculosis. J. Dairy Sci. 91:4599-4609.

Kudahl, A. B., S. Østergaard, J. T. Sørensen, and S. S. Nielsen. 2007. A stochastic model simulating paratuberculosis in a dairy herd. Prev. Vet. Med. 78:97-117.

Littell, R. C., G. A. Milliken, W. W. Stroup, R. D. Wolfinger, and O. Schabenberger. 2006. SAS for Mixed Models. 2nd ed. SAS Institute Inc., Cary, NC.

Manning, E. J. B., and M. T. Collins. 2001. Mycobacterium avium subsp. paratuberculosis: Pathogen, pathogenesis and diagnosis. Rev. Sci. Tech. Off. Int. Epizoot. 20:133-150.

McKenna, S. L., G. P. Keefe, H. W. Barkema, J. McClure, J. A. Vanleeuwen, P. Hanna, and D. C. Sockett. 2004. Cow-level prevalence of paratuberculosis in culled dairy cows in Atlantic Canada and Maine. J. Dairy Sci. 87:3770-3777.

Nielsen, S. S. 2002. Variance components of an enzyme-linked immunosorbent assay for detection of IgG antibodies in milk samples to Mycobacterium avium subsp. paratuberculosis. J. Vet. Med. B 49:384-387.

Nielsen, S. S. 2007. Danish control programme for bovine paratuberculosis. Cattle Pract. 15:161-168.

Nielsen, S. S. 2008. Transitions in diagnostic tests used for detection of Mycobacterium avium subsp. paratuberculosis infections in cattle. Vet. Microbiol. 132:274-282.

Nielsen, S. S., B. Kolmos, and A. B. Christoffersen. 2004. Comparison of contamination and growth of Mycobacterium avium subsp. paratuberculosis on two different media. J. Appl. Microbiol. 96:149-153.

Nielsen, S. S., M. A. Krogh, and C. Enevoldsen. 2009. Time to the occurrence of a decline in milk production in cows with various paratuberculosis antibody profiles. J. Dairy Sci. 92:149-155.

Sweeney, R. W., R. H. Whitlock, S. McAdams, and T. Fyock. 2006. Longitudinal study of ELISA seroreactivity to Mycobacterium avium subsp. paratuberculosis in infected cattle and culturenegative herd mates. J. Vet. Diagn. Invest. 18:2-6.

Whitlock, R. H., L. T. Hutchingson, R. Merkal, L. T. Glickman, C. Rossiter, S. Harmon, P. Spencer, J. Fetrow, J. Bruce, C. E. Benson, and J. Dick. 1985. Prevalence and economic consideration of Johne's disease in the northeastern U.S. Proc. US Anim. Health Assoc. 89:484-488. 
APPENDIX

Table A1. Final model estimates of the analysis of data set 1 with slaughter weight as the outcome variable

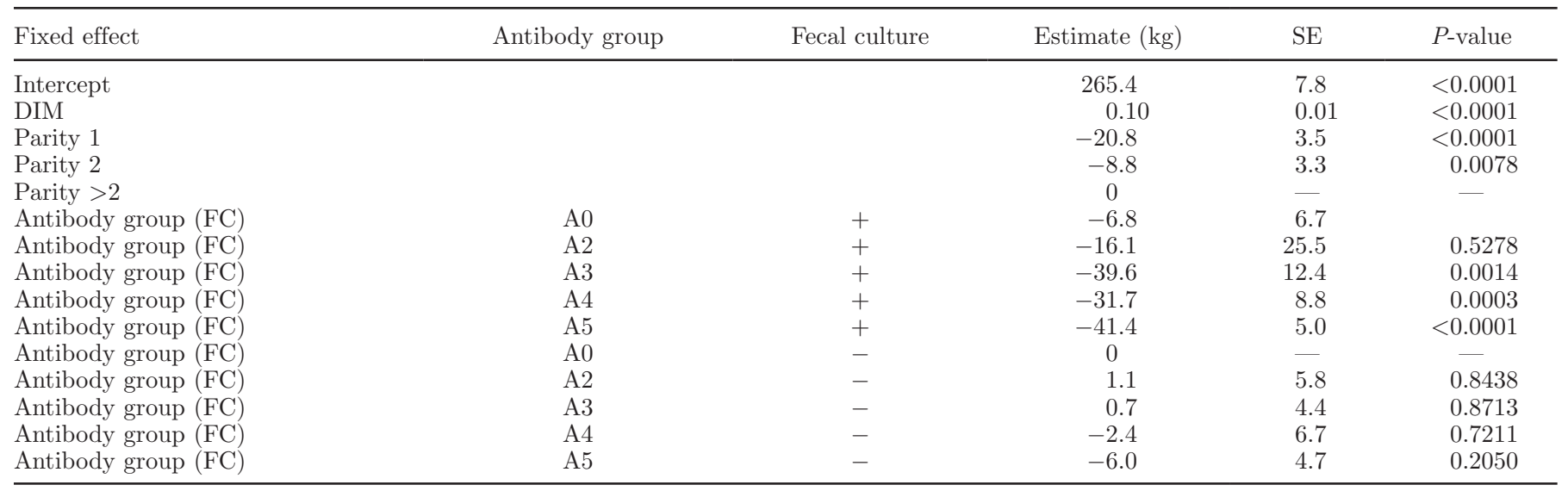

${ }^{1}$ Only Danish Holsteins were included in this analysis. Antibody group was nested within fecal culture (FC).

Table A2. Effect of antibody group and fecal culture (FC) response on slaughter value ${ }^{1}$

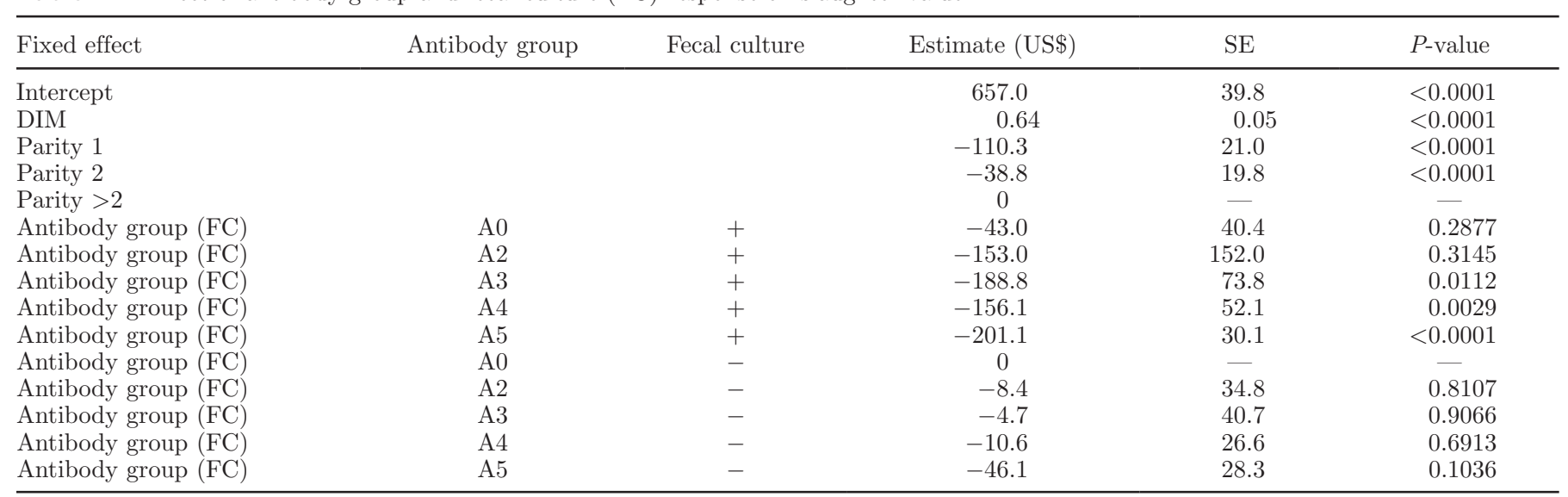

${ }^{1}$ Resulting model estimates of the analysis of data set 1. Only Danish Holsteins were included in this analysis. Antibody group was nested within FC. 